\title{
Birgit Althans
}

„Halte dich fern von den klatschenden

Weibern ..."

Zur Phänomenologie des Klatsches

Der Klatsch gilt als Negativ-Form eines ,vernünftigen Gesprächs', als leeres Geschwätz, als sinnloses Geplauder und in dieser Negativfunktion auch als ,typisch weiblich‘. Ich möchte in diesem Aufsatz den Versuch machen, dieses Negativbild als Positiv zu entwikkeln, es - um bei diesem Bild zu bleiben - als (Dia)positiv anders zu beleuchten. Dabei nutze ich vor allem die Etymologie des Klatschbegriffs und unterstütze deren Wirkung durch volkskundliches Material und Literatur des Frauenbildes des 19.Jh. Als Projektionsapparat dienen die aufhellenden Theorien Elias' und Foucaults über die zivilisationsbedingten Entwicklungen bestimmter Affekte.

\section{Zur Etymologie des Klatsches}

Wie konnte ein ursprünglich onomatopoetisches Wort zu seiner Bedeutung als verletzendes Gespräch über das Intimste abwesender Personen avancieren? In der etymologischen Recherche' ${ }^{\prime}$ offenbart sich uns die heute nächstliegende Bedeutung , abwertendes Gerede über Abwesende‘ als eine Zuschreibung der bürgerlichen Gesellschaft. Die Wortwurzel, der mittelhochdeutsche Iterativ ,klaz', beschrieb bis zum 18. Jh. entweder einen ,schallenden Schlag' oder einen ,feuchten Fleck', ,Schmutzfleck'. Gleichzeitig mit der negativen Bedeutung des Klatsches als, üble Nachrede' entstand die diffamierende Zuordnung dieser Handlung als eine ,typisch weibliche‘ Gesprächsform, die schließlich fast zu einer geschlechtsspezifischen Eigenschaft wird. Dies wird in der Ableitung, Klatsche' (= Peitsche, Fliegenklatsche, , Frau) sowie den Benennungen ,Klatschtante', ,-base', ,trien“ sehr deutlich. In den Ausdrücken ,Klatschfutter', ,-kasten', ,-dose‘, ,-loch“, die sich sehr deutlich auf das weibliche Geschlechtsorgan beziehen, ist die Zuordnung unverkennbar. ${ }^{2}$ Die Untersuchung der Redewendungen, die die Klatschhandlung ebenfalls synonymisch beschreiben, wie z.B. ,schmutzige Wäsche waschen', ,jemanden durchhecheln' oder ,jemandem am Zeug flicken', weisen deutlich auf Orte kollektiv verrichteter Frauenarbeit hin, vor allem auf die Waschplätze, die früher gefürchtete Klatschorte gewesen sein müssen. 


\section{II. ,A Wasch un ä Klatsch ist bald gemacht'}

Das dokumentieren Sprichwörter wie „Wann a ding auf die waschbanck, in die badstub und für den backofen kommt, so ists weit gnu auskommen“3, denn ,Wenn die Weiber waschen und backen, haben sie den Teufel im Nacken".

Männer, die an dieser durch den Arbeitsvorgang bedingten ,in-group' allenfalls als ferne Beobachter teilhaben konnten, schienen gleichwohl eine starke Identifikation mit dem manuell und verbal bearbeiteten Gegenstand vorzunehmen.

Die Waschbleuel, mit denen die eingeweichte Wäsche lange mit ,schallenden Schlägen* bearbeitet wurde, dienen auf so manchen karikierenden Darstellungen als weibliche Waffen im berüchtigten Kampf um die Hosen. Und die waschenden Frauen wußten ihn wohl zu gebrauchen. So konstatiert Lindenborn 1742 in seinem „Diogenes“, daß „... waschweiber . . . auf den beiden Hintervierteln der gewesenen atzel so emsig mit den Waschbleueln gleichsam den Schmieds-dreyschlag hielten". 4 Nicht minder gefürchtet war die verbale Schlagkraft der weiblichen Zunge, die sich ebenfalls in so manchem Sprichwort wie „ein bös' Weib führt die Peitsche im Maul, damit sie den Mann geiszelt“ oder auch „Weiberzunge ist eine Schere, bald schneidend, bald stechend, bereit zur Wehre "5 dokumentiert. Ich habe, um dem näherzukommen, was beim Waschen tatsächlich geäußert wurde, einige spezifisch weibliche Arbeitslieder, deren Melodie auf dem Rhythmus von schallenden Schlägen basiert, zu Rate gezogen. Da sind zunächst die Lieder lettischer Frauen, die den direkten Bezug des Gesprächs zum bearbeiteten Gegenstand bestätigen und auch offen geäußerte sexuelle Wünsche beinhalten.

Hoch schwinge ich den Wäschestock über meinem Kopfe, wenn ich die Burschenhose wasche, und denke: hätt' ich doch den Stock, der sonst in diesen Hosen sitzt.

Aber auch negative Erfahrungen mit der Sexualität werden deutlich:

Meine Mutter sagt, sie gibt mich einem, der Erbarmen mit mir hat.

Nur, was heißt denn hier Erbarmen?

Er spießt den Dolch mir in den Bauch. ${ }^{6}$

Auch die schottischen waulking songs ${ }^{7}$ - waulking = schottisch für walken - , ausschließlich von Frauen gemachte Lieder, deren Entstehungsgeschichte bis ins 16. Jh. zurückverfolgt werden kann, dokumentieren die weibliche Sicht des täglichen Lebens, unerfüllte Liebe, ungewollte Schwangerschaften und Erlebnisse wie diese:

Eines Tages ging ich durchs Moor

Ich traf niemand außer Donald

Wir begannen spielerisch zu flirten

Das Flirten entwickelt sich zur Angst

Er drohte mir mein Hemd in Fetzen zu reißen

Er trat meine Haare mit seinen Schuhen

Er nahm ein kleines Messer aus seiner Tasche

Das war nicht das, was du mir versprochen hast:

Eine Verlobung, und ein Hochzeitsfest und eine Trauung ${ }^{8}$.

In dem Material, das zusammen mit den Songs herausgegeben wurde, finden wir auch die Darstellung des waulkings von einem englischen Reisenden des 18 . Jh. ${ }^{9}$. Er beschreibt 
das waulking als, ohrenbetäubenden Lärm', die arbeitenden Frauen als, entfesselte Furien', für die er eine Einweisung nach Bedlam, in die damals berüchtigte Irrenanstalt, empfiehlt. Der Blick dieser Reisenden scheint mit exemplarisch zu sein für die Veränderung der Wahrnehmungsstrukturen im Zuge der industriellen Revolution: Die ungehemmte, lustvolle Atmosphäre dieser Frauenöffentlichkeiten wird als bedrohlich erlebt und in den Kontext des Anormalen ver-rückt.

Mit ähnlichem Mißtrauen werden bis ins 20. Jh. - zumindest in Frankreich - die öffentlichen Waschhäuser für proletarische Frauen betrachtet, Orte, ... . . wo auch der Gewalttätigkeit freier Lauf gelassen wird, zum Entsetzen jener, die im Namen der Wohlanständigkeit den Frauen das Recht auf Zornausbrüche, Schreie und Prügeleien absprachen. Es ist auch ein Ort des Konflikts zwischen den Frauen und der Staatsmacht, die an solchen Ausbrüchen und mehr noch an der Zeitvergeudung der Frauen AnstoB nimmt. " ${ }^{10} \mathrm{Ge}$ fürchtet waren sie ebenfalls in ihrer Funktion als Orte sozialer Kontrolle, da die Beschäftigung mit der Leibwäsche, in der sich der ganze Körperschmutz absetzte und die für sich schon eine indiskrete, die Intimsphäre berührende Sprache sprach, das Gespräch über körperlichen Schmutz und mangelnde Reinlichkeit, über mögliche Schwangerschaften und sexuelle Kontakte bedingte.

So beschreibt Zola die Kommentare der großen Clémence im Waschhaus beim Sortieren der Wäsche.

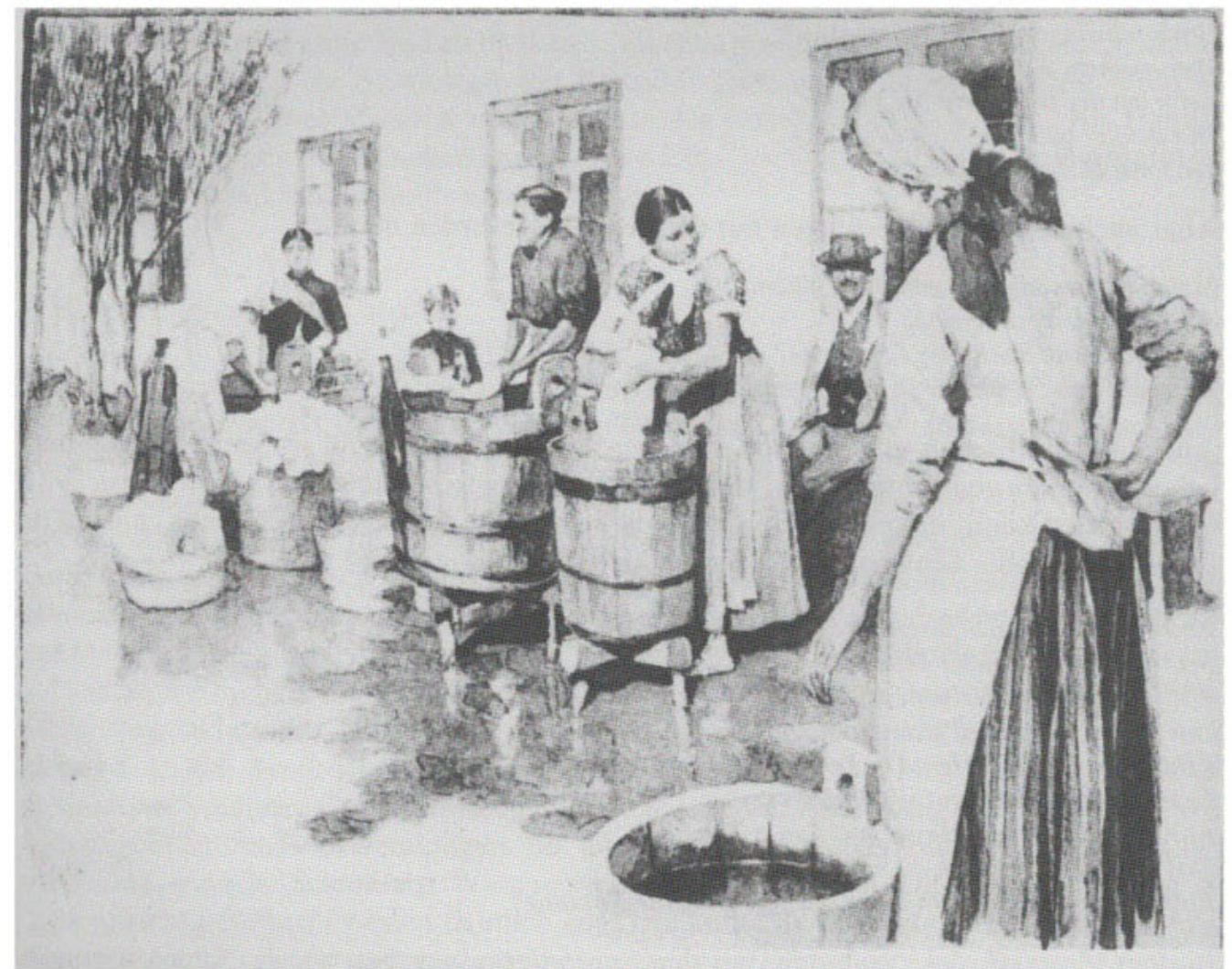

In der Wäscherburg. Nach einem Aquarell von F.v. Myrbach, 1895 
„Da ließ diese lange nichtsnutige Person bei jedem Stück eine derbe Bemerkung, eine Zote vom Stapel; sie breitete das Elend der Kunden, die Erlebnisse der Alkoven aus: Sie hatte für alle Löcher und alle Flecken, die ihr durch die Hand gingen, einen Werkstatscherz bereit. Augustine tat so, als verstehe sie nicht, und sperrte ihre Ohren, die Ohren eines kleinen, lasterhaften Mädchens, weit auf. [. . . J So wurde das ganze Viertel La Goutte-d'or bei jedem Wäschesortieren entkleidet. "

Ebenfalls berühmt-berüchtigt war die zotenreiche Sprache der Wäscherinnen, die oftmals eine soziale Funktion gehabt zu haben scheint. So hatte in einem spanischen Dorf das Ausharren am Waschplatz die Bedeutung eines Initiationsritus für die männlichen Dorfbewohner in ihre Männerwürde.

„Paco von der Mühle ging dorthin schwimmen (es handelt sich um einen Teich, an dessen Ufer gewaschen wurde) und setzte sich mit großem Vergnügen mehr als zwei Stunden lang den Scherzen der Wäscherinnen aus. Sie riefen ihm etwas zu, um ihn zu provozieren, machten für Frauen typische Bemerkungen, um ihm zu schmeicheln. All das stellten quasi Initiationsriten dar. Von diesem Zeitpunkt an ließen ihn seine Eltern abends allein ausgehen, und er mußte auch nicht nach Hause kommen, bevor sie ins Bett gingen." 12

Yvonne Verdier geht davon aus, daß die zotigen Beschimpfungen Schutzfunktionen hatten und als Abwehrmechanismen verstanden werden können.

„Vom Schmutz des Körpers ist es zum Schmutz der Seele nicht sehr weit. Gemeinsam seine schmutzige Wäsche zu waschen oder die schmutzige Wäsche der anderen, bedeutet in jedem Fall, die Nase in die Angelegenheiten anderer zu stecken, in die Geheimnisse ihrer Not, ihrer Gedanken, ihrer Wünsche, ihrer Seele . . e einzudringen, aber gleichzeitig entfernt man Flecken und Schmutz, macht alles wieder rein." 13

Diese Möglichkeit, auf diese Art ,schmutzige Wäsche zu waschen‘, verliert sich mit der Durchsetzung der bürgerlichen Lebensweise.

\section{Die Entstehung des müßigen Geplauders}

Im Zuge der Konsolidierung der Trennung zwischen öffentlicher und privater Sphäre und der damit verbundenen Ausgrenzung der bürgerlichen Frau aus der Öffentlichkeit in die abgeschlossene Intimspäre des ,trauten Heims، werden nicht nur die traditionellen Frauenöffentlichkeiten der Waschplätze für die bürgerliche Frau tabuisiert, sondern auch das Gespräch über intime Belange wird ver-heimlicht.

„Der Leib und alles, was damit assoziiert wird, hatte von nun an im Verborgenen zu bleiben. Hatte nicht Kant die Menschen von der bürgerlichen Öffentlichkeit ausgeschlossen, die nichts anbieten konnten als Eigentum als ihren, Leib', ihr, leibhaftiges' Arbeitsvermögen? Der Bürger schuf sich heimliche Winkel für seine, Notdurft': Schlafzimmer, Klosett- und die Kanalisation, die ihn unterirdisch mit anderen Bürgern verband. . . Auch die schmutzige Wäsche sollte nicht mehr in der Öffentlichkeit gewaschen werden. Mit der Kanalisation kam das Wasser ins Haus der Bürger, wie später Gas und Strom, aus unsichtbaren Quellen, die nicht zu versiegen schienen. "14

Als ein gleichermaßen unsichtbaren Kanalisationssystem, das den nunmehr verheimlichten Körper- und Seelenschmutz aufnahm und von Haus zu Haus weitertransportierte, kann der Klatsch der bürgerlichen Frauen aufgefaßt werden: als murmelnder und tuschelnder Gesprächsfluß vor allem über sexuelle Belange hinter den Fassaden der Bürger(-häuser). Foucault beschreibt die Tabuisierung des Sprechens über Sexualität als Auslöser einer diskursiven Explosion: 
„Wohlverstanden: Es mag schon sein, daß eine Säuberung - und zwar eine unerbittliche Säuberung - des zugelassenen Vokabulars stattgefunden hat. Es mag auch sein, daß man eine ganze Rhetorik der Anspielung und der Metapher kodifiziert hat. Zweifellos haben neue Regeln des Anstands die Worte gefiltert: Polizei der Aussagen. Und es gab auch eine Kotrolle des Außerungen: man hat in sehr viel strengerer Weise festgelegt, wo und wann, in welcher Situation, zwischen welchen Gesprächspartnern und innerhalb welcher gesellschaftlichen Beziehungen es möglich war, von Sex zu sprechen; auf diese Weise hat man Bereiche geschaffen, in denen zwar kein absolutes Schweigen, wohl aber Takt und Diskretion herrschten: zwischen Eltern und Kindern zum Beispiel oder zwischen Herren und Bediensteten. " 15

Die bürgerliche Frau etabliert ebenfalls einen Bereich, in dem über Sexualität gesprochen werden konnte: den Kaffeeklatsch. Die Kaffeekränzchen werden von der Männergesellschaft als ,Abklatsch` der für die bürgerliche Männeröffentlichkeit konzipierten Kaffeehäuser belächelt, wie auch der ,Kaffeeklatsch“ als Karikatur des Kaffeehauses belächelt wird.

Mir scheint es sich bei dieser intimen Öffentlichkeit jedoch weniger um einen Abklatsch der Männerkultur als vielmehr um eine sublimierte Fortführung der Tradition der Waschplatzöffentlichkeiten zu handeln. Wobei das Wort ,Klatsch' nunmehr die verbale Schlagfertigkeit zu bezeichnen scheint, mit der die Flecken aus der scheinbar weißen Weste des Gesprächsgegenstandes herausgeklopft werden. Auch Jean Paul scheint auf die Waschplatzöffentlichkeiten zu rekurieren, wenn er eine Dame beschreibt, die ... . die großen künstlichen Waschmaschinen, in welchen ganze Familien auf einmal (thee oder kaffee wird als Lauge zugegossen) sehr gut eingeweichet, gehandhabt und gewalket werden, niemals in ihrer Stube auftreten (ließ). . . “16

Das ,schmutzige Wäsche waschen' war komplizierter geworden. Die verräterischen Flecken in der Leibwäsche kamen den am Kaffeeklatsch beteiligten Damen nicht mehr vor Augen. Statt dessen nimmt das Frauengespräch die Bedeutung auf, die das äußere Erscheinungsbild als Statusrepräsentanz der Persönlichkeit gewinnt. Die Kleidung wurde gerade in einer Zeit, in der das Erscheinungsbild der Bürger der Neutralität verpflichtet war, zu einem wichtigen Indiz der Entschlüsselung der Persönlichkeit. Erkennt man die Kleidung eines Gentleman daran, daß die Knopflöcher am Ärmel seines Mantels aufknöpfbar sind, sein Verhalten aber daran, daß er diese Knöpfe stets sorgfältig zuknöpft, um keine Aufmerksamkeit auf seine Ärmel zu lenken, so macht eine Dame, die keine ist, dies durch einen um eine Nuance zu langen Blick und mit einem unmerklich hochgezogenen Rocksaum deutlich.

„Spürsinn war erforderlich, um eine Beziehung zur Person hinter der Fassade ihrer äußeren Erscheinung herzustellen. Wer jedoch die Regeln, die die jeweilige äußere Erscheinung bestimmten, nicht kannte, wer den Kravattenknoten oder das Tuch über dem Chignon nicht zu ,lesen" verstand, der konnte auch keine Gewißheit darüber gewinnen, mit wem er es auf der Straße zu tun bekam. "17

Einer ehrbaren Dame geriet unter diesen Bedingungen das Auftreten in der Öffentlichkeit zur Tortur, die sie mit eisigster Zurückhaltung bewältigte. Gleichzeitig wuchs jedoch die Gier, die Persönlichkeit - von der eigenen Maske zurückgehalten - hinter den Fassaden der anderen zu entlarven.

Vor allem hinter der Fassade anderer Frauen, die den gleichen Verhaltensnormierungen unterworfen waren, wurden die unglaublichsten Begierden vermutet. So erklärt sich auch der Boom der englischen Sensationsliteratur im 19. Jh., wo die viktorianische Dame sich mit den verführerischen weiblichen Hauptfiguren identifizierte und durch gieriges Verschlingen ihre geheimen Bigamie-, Ehebruch- und Gattenmordgelüste befriedigte. ${ }^{18}$ Und so erklärt sich auch die Lust am Klatsch. Beim Kaffeeklatsch bestand die Möglich- 
keit, eng geschnürt ins Korsett der eigenen Ehrbarkeit, durch das Debattieren über die Verstöße anderer Frauen gegen die Gebote der Schicklichkeit, die eigenen Übertretungsund Ausbruchsphantasien zumindest verbal zu berfriedigen - und sie gleichzeitig entrüstet $\mathrm{zu}$ verdammen.

Die zunehmende Unfähigkeit, über das - mit Scham- und Reinlichkeitsschwellen verschlossene Innere zu sprechen, produziert den verschlüsselten Klatschcode und die oft hämische Lust, das, was man bei sich nicht wahrnehmen darf, bei anderen zu fixieren und ausführlich zu diskutieren. Der ,Takt ${ }^{\star}$, mit dem nun - schon lange abgelöst vom ursprünglichen Rhythmus der Arbeitshandlung - geklatscht wurde, um die Gesprächsharmonie zu bewahren, war bewundernswert. Er war das Ergebnis einer langen Schulung, in Gesprächen die leisesten Nuancierungen im Tonfall wahrzunehmen und hinter dem bedeutungsvollen Schweigen die gemeinte Anspielung zu erahnen. Sexuelle Erfahrungen wie Vergewaltigung oder Frigidität wurden als Geschichten über andere Frauen verpackt und so vermittelt. Abtreibungsadressen wurden unter dem Mantel von Formulierungen wie „Ich habe von einer Schauspielerin gehört, die ja zu . . ."weitergegeben. Dabei war gewiß, daß die wahren Motive der Sprecherinnen in der Gesprächssituation nicht aufgedeckt wurden - man sprach ja über andere!

Dieser Anspielungscode, der sich im weiblichen Gespräch zunehmend etabliert, muß im Kontext des allgemeinen Umgangs mit der Sexualität begriffen werden. Foucault beschreibt, daß parallel zur privaten Tabuisierung des Gesprächsthemas Sexualität eine Vermehrung des öffentlichen Interesses an der Sexualität angeregt wird.

„.. . man muß vom Sex sprechen wie von einer Sache, die man nicht einfach zu verurteilen oder zu tolerieren, sondern vielmehr zu verwalten und in Nützlichkeitssysteme einzufügen hat, einer Sache, die man zum größtmöglichen Nutzen aller regeln und funktionieren lassen muß. Der Sex, das ist nicht nur eine Sache der Verurteilung, das ist eine Sache der Verwaltung. Es ist Sache der öffentlichen Gewalt, er erfordert Verwaltungsprozeduren, er muß analytischen Diskursen anvertraut werden . . "19

Der weibliche Körper und die Sexualität werden im Kontext der neu entstandenen ,politischen Ökonomie der Bevölkerung، zu einem Gegenstand staatlicher Kontrolle.

Um seine Gebärfunktion zu erhalten und möglichen Krankheiten vorzubeugen, entstand im 18. Jh. ein intensiver Hygienediskurs, der sich in Hygienezeitschriften für die Frau niederschlägt, und der das Ziel eines gesunden Körpers in die Erhaltung eines reinen Körpers und - reiner Gedanken transformiert.

„Das Ziel der Reinheit läßt nur eines zu: die Reinigung, sowohl der Körper wie der Gedanken. Die Reinigung kommuniziert mit der Gefährdung und sucht sie zu bannen. “20 Aber „Der Zustand der Reinheit hat immer nur vorläufigen Charakter, zumal der Schmutz sich dadurch auszeichnet, daß er immer noch tiefer liegt, als der, der schon sichtbar gemacht und weggesäubert wurde. Wo Reinigung und Schmutz Hand in Hand gehen, ist der Gedanke der Grenze strukturell unmöglich geworden. Wer weiß angesichts des Schmutzes im Innern der Körper schon genau, ob der Tiefenlöser die Tiefen auch wirklich erreicht." $" 21$

Dieser tief im eigenen Innern vermutete Schmutz scheint im Unbewußten oder UnterBewußten zu gären und unreine Gedanken zu produzieren, er verlangt ein Ventil, um entweichen zu können - und findet es im Klatsch. Auch wenn es keine traditionellen Frauenöffentlichkeiten mehr gibt, wenn das müßige Geplauder als kleiner Schwatz während zunehmender Hektik auf dem Weg vom Büro zum Kopierer oder während des Einkaufens vor den Ladeneingängen stattfinden muß, auch wenn es vornehmlich von der Regenbogenpresse annektiert und pervertiert wird, auch wenn auch Männer klatschen, erfüllt der Klatsch weiterhin seine Funktion als - wenn auch flüchtiges - Solidarisierungsinstrument einer in-group und ermöglicht eine Aggressionsabfuhr in tolerierter, kanali- 
sierter Form. Zudem gilt er immer noch als ,typisch weibliche' Kommunikationsform, was sich auch in der Verdammung schwatzhafter Männer mit den Benennungen ,Klatschtante', ,-base' ausdrückt. Liegt in dieser Verurteilung der weiblichen Rede - es sei angemerkt, daß noch immer sehr gern jegliches weibliches Sprechen mit dem Klatschen gleichgesetzt wird - , die als banales, triviales Geschwätz, Reden über Nichtigkeiten, leeres, oberflächliches Gerede gilt, nicht doch ein heimliches Unbehagen? Dazu Adorno:

„Für den Begriff der Tiefe folgt . ., daß tief sei, was die Erfahrung des Negativen, was das Leiden in irgendeiner Weise bejaht, während alle anderen Anschauungen platt sein sollen, die ihm sich entgegenstellen. . . Ich kann dieser Genese auch dieser Denkart nicht ausführlich nachgehen, sie hängt zweifellos mit der Diffamierung der Sinne und schließlich mit dem in unserer gesamten Zivilisation geltenden Sexualtabus zusammen und ist, wie Nietzsche bis ins einzelne ausgefühn hat, reaktiv. Das heißt, was man sich unter dem Zwang der Ordnung verbieten muß, das macht man zu einem an und für sich Schlechten, Oberflächlichen, Banalen und Trivialen. "22

Für Männer ist das Gespräch von Frauen über ihre Sexualität eine Entmystifikation ihrer - heimlichen - Vorstellung. Ein Mann sieht, wie Claudia Gehrke schreibt “. . . bei der Frau das Ungesehene, etwas, das er nicht sieht, wenn es ihn berührt. Das Geheimnis. Um die Scham von sich abzuwenden, erfindet er die Scham der Frau. Doch kann er seine verlieren? Seine Heimlichkeit wird zur Unheimlichkeit, wenn er sie nicht veröffentlicht, wird seine Macht möglich. “23

In der Diffamierung des Frauenklatsches könnte sich somit die männliche Scham verberben. Aber ich wahre den Takt.

\section{Anmerkungen}

1 Die etymologischen Ergebnisse beruhen auf Studien der Wörterbücher: Jakob u. Wilhelm Grimm, Deutsches Wörterbuch, Bd. 11, Bd. 39, Leipzig 1873 und 1922; Moritz Heyne, Deutsches Wörterbuch, Leipzig 1892; Friedrich Kluge u. Alfred Götze, Etymologisches Wörterbuch der deutschen Sprache, Berlin 1951; Duden, Bd. 7, Etymologie. Mannheim 1963.

2 Interessant ist an dieser Stelle die Arbeit von Luise Frei über die Gleichsetzung der Benennungen von Frauen mit der Gefäßform ihres Geschlechts. Vgl. Luise Frei (Dissertation), Die Frau in der schweizerischen Volkssprache, Zürich 1935, 22.

3 vgl. Karl Friedrich Wander, Deutsches Sprichwörterlexikon, Bd. 1-5, Leipzig 1867.

4 zit. n. Grimm, Bd. 39, a.a.O., 2217.

$5 \mathrm{vgl}$. Wander, Sprichwörterlexikon, Leipzig 1867. Bd. 5, 16.

6 zit. n. Bud Berzing (Hrsg., Sex songs of the ancient letts, University Books, New York 1969, S. 199. S. 245.

7 Bei dem waulking handelt es sich um einen den Frauen vorbehaltenen ProzeB der Tweedherstellung auf den Hebriden, der bis Ende des zweiten Weltkriegs existierte; bei meiner Suche, nach
Frauen-Arbeitsliedern, die auf Klatschrhythmus basieren, bin ich darauf gestoßen. 6-14 Frauen sitzen um einen Tisch versammelt und lassen ein zu einem Ring zusammengerolltes zusammengeknüpftes Stück Tweed unter ihren schlagenden Händen um den Tisch rotieren. Gesungen wird, um der Ermüdung vorzubeugen und um einen einheitlichen Rhythmus zu garantieren. Am Ende des waulkings, nach ca. 3 Stunden, ist der Gesang der Frauen wesentlich lauter, enthemmter.

8 zit. n. Helen Creighton/Calum Macleod (Hrsg.), Gaelic Songs in Nova Scotia, Ottawa 1964, song No. 69.

9 vgl. J.L. Campbell/Francis Collinson (Hrsg.), Hebridean Folksongs, Oxford 1969, 4 ff.

10 zit. n. Michelle Perrot, „Rebellische Weiber. Die Frau in der französischen Stadt des 19. Jahrhunderts." in: Claudia Honegger/Bettina Heintz (Hrsg.). Listen der Ohnmacht. Frankfurt 1981, S. $71-99 ; 87$.

11 zit. n. Emile Zola, Der Totschläger, München 1965.

12 zit. n. Yvonne Verdier, Drei Frauen. Das Leben auf dem Dorf, 1982, 138.

13 Ebd. 140. 
14 Ilona Ostner, „Frauen und Offentlichkeit ${ }^{4}$, in: Arch + 60, 1981, 28.

15 Michel Foucault, Sexualität und Wahrheit, Ffm. 1983, 27.

16 Jean Paul, „Das heimliche Klagelied der jetzigen Männer", in: Sämtliche Werke, Bd. 39, Berlin $1829,9$.

17 zit. n. Richard Sennett, Verfall und Ende des offentlichen Lebens und die Tyrannei der Intimität, Ffm. 1983, 195.

18 Elaine Showauter, „Ausbruchsphantasien, Ehebruch, Mord und Bigamie in der englischen Tri- vialliteratur", in: Listen der Ohnmach, a.a.O. 259-276.

19 Foucault, a.a.O., 36.

20 Christa Karpenstein-EBbach, „Saubere Weiber authentische Frauen ", in: Konkursbuch 12, Tübingen 1984, 152.

21 Ebd. 153.

22 Theodor Adorno, Philosophische Terminologie, Ffm. 1973, 171-172.

23 Claudia Gehrke, „Heimlichkeit-Geheimnis-Gewalt", in: Konkursbuch 12, Tübingen 1984, $141-142$. 